\title{
Reformar para privatizar la educación superior en Brasil ${ }^{1}$
}

\author{
França, Eliacir Neves \\ Universidade Estadual de Londrina, Londrina, Brasil \\ eliacir@uel.br \\ Aguilar, Luís Enrique \\ Universidade Estadual de Campinas, Campinas, Brasil \\ luis.aguilar@merconet.com.br \\ Afonso, Almerindo Janela \\ Universidade do Minho, Braga, Portugal \\ ajafonso@uminho.pt
}

\section{Resumen}

La educación superior se convirtió a partir de mediados del siglo pasado un espacio estratégico para la consolidación del proyecto político, económico y financeiro del grupo dominante de la actualidad. En 1995, cuando se dio la celebración del «Acuerdo General sobre el Comercio y los Servicios» (GATS), el mismo año que entra en vigor oficialmente la Organización Mundial del Comercio (OMC), la educación se introdujo en el rol de servicios, pasando desde entonces a ser entendida como una mercancía. El GATS fue el primer instrumento de aplicación multilateral y universal en este sector, favoreciendo la liberalización global del comercio de servicios. A partir de este acuerdo la formación ofrecida por instituciones de educación superior es transformada en una mercancía regulada por la lógica del lucro y de la acumulación. La reforma en los sistemas de educación superior fue recomendación de la OMC con el fin de reducir costos e incrementar una mayor autonomía para las instituciones, esta entendida claramente como la tarea preponderante de buscar otras fuentes de financiamiento a través de alianzas con el sector productivo, privatización del sector. En Brasil, esta recomendación fue seguida por la vía de la institucionalización de fundaciones en el interior de las universidades públicas y por el aumento del número de instituciones privadas. Como miembro de la OMC, Brasil, en el mandato del ex presidente FHC (1995 - 2002) emprendió una profunda reestructuración del Sistema Nacional de Educación Superior. El proyecto de reforma de este nivel de enseñanza llevó al enjuagado de las universidades públicas con cortes radicales en las inversiones y, también, con la transferencia de la actividad de oferta de cursos y programas de educación superior al sector privado. Resultado: se privatizó el sector contribuyendo a la exclusión de miles de jóvenes que solo volver a las universidades en el Gobierno Lula (2003 - 2011). Este cambio está hoy profundamente amenazado.

\section{Abstract}

Higher education became, from the middle of the last century, a strategic space for the consolidation of the political project of the dominant group of today. In 1995, when the General Agreement on Trade and Services (GATS) was concluded, the same year that the World Trade Organization (WTO) officially entered into force, education was included in the list of services, , to be understood as a commodity. The GATS was the first instrument of multilateral and universal application in this sector favoring the global liberalization of trade in services. From this agreement the training offered by institutions of higher education is transformed into a commodity regulated by the logic of profit and competition. The reform in higher education systems was a recommendation of the WTO to reduce costs and increase the autonomy of institutions, which is clearly understood as the primary task of seeking other sources of financing through partnerships with the productive sector, privatization of the sector. In Brazil, this recommendation was followed by the institutionalization of foundations within the public universities of the federal system and by the increase in the number of private institutions of higher education. As a member of the WTO, Brazil under the mandate of former President FHC (1995-2002) undertook a profound restructuring of the National System of Higher Education. The project to reform this level of education has led to the reduction of public universities with radical cuts in investment and also to the transfer of the activity of offering courses and higher education programs to the private sector. Result: the sector was privatized contributing to the exclusion of thousands of young people who only return to the universities in the Lula government.

Palabras clave: educación superior; exclusión educativa, políticas públicas, privatización; mercado educativo.

Keywords: higher education; educational exclusion, public policies, privatization; market. 


\section{IINTRODUCCIÓN}

La educación superior se ha convertido a partir de mediados del siglo pasado un espacio valioso para la consolidación de un proyecto político que representa los intereses de la clase hegemónica. (Barreto; Leher, 2008).

En la década de 1970, Martin Trow, profesor de la Universidad de California, propone un modelo que intenta explicar el desarrollo de los sistemas de educación superior, clasificándolo en tres fases: sistema de elite, sistema de masa y sistema universal. (Trow, 1973).

El «sistema (1) de élite - realiza la función de dar forma a la mente y el carácter de una clase dirigente y prepararlos para puestos de liderazgo; (2) masa - transmisión de competencias y preparación para una amplia gama de funciones técnicas ante la élite económica; y (3) universal - adaptación del conjunto de la población al rápido cambio social y tecnológico «. (Trow, 2005, p.1) (Traducción nuestra).

La transición entre cada una de las fases anteriores demanda cambios en diferentes aspectos de las estructuras y funciones de las instituciones de educación superior. Entre ellos podemos citar: el tamaño del sistema, el alcance de la educación, el currículo, la diversificación de la estructura organizativa, formas de acceso y selección y modelos de gestión. A lo largo de los siglos esta transición se ha ligado, fundamentalmente, al modo de producción de cada período histórico. Esto demuestra que la educación superior se constituye en un sector estratégico por involucrar la formación de las élites, de los técnicos y de la mano de obra para el mercado de trabajo.

En la Ronda de Uruguay, en 1995, cuando se dio la celebración del «Acuerdo General sobre el Comercio y los Servicios» (GATS), el mismo año que entra en vigor oficialmente la Organización Mundial del Comercio (OMC), la educación se inserta en el rol de los servicios, pasando, desde entonces, a ser entendida como una mercancía.

La OMC es una OI, cuyo objetivo es favorecer y regular el comercio entre los países miembros en las más diversas áreas y cuestiones. La actuación de esta institución no se restringe al comercio de bienes materiales. Así, actúa en «áreas antes no reguladas por criterios comerciales y mercantiles, tales como los servicios educativos y los relacionados con los derechos de propiedad intelectual». (Borges, 2009: 84).

EI GATS fue el primer instrumento de aplicación multilateral y universal en este sector, favoreciendo la liberalización global del comercio de servicios. A partir de este acuerdo la formación ofrecida por instituciones de educación superior es transformada en una mercancía regulada por la lógica del lucro y de la competición.

La concepción de educación superior de la Organización Mundial del Comercio (OMC) - World Trade Organization (OMC) - se encuentra formulada en los documentos Acuerdo General sobre el Comercio de Servicios (AGCS) - General Agreement on Trade in Services (GATS) (1995) , Servicios de Enseñanza - Nota Documental de la Secretaría (1998), Comunicación de los Estados Unidos (2000), Comunicación de Australia (2001), Comunicación de Japón (2002). Estos documentos se constituyen en referencias discursivas protagonizadas por la OMC y / o en el marco de esta última, como es el caso de las comunicaciones realizadas por los países miembros de dicha organización internacional. (Borges, 2009: 84).

La reforma en los sistemas de educación superior fue recomendación de la OMC con el fin de reducir costos e incrementar una mayor autonomía para las instituciones, esta entendida claramente como la tarea preponderante de buscar otras fuentes de financiamiento a través de alianzas con el sector productivo. En las instituciones públicas, en Brasil, esta recomendación fue seguida por la vía de la institucionalización de fundaciones en el interior de las universidades públicas del sistema federal. De esta forma el aspecto más caro para las instituciones universitarias, su autonomía se ha reducido al aspecto de gestión financiera y administrativa. La didáctico-científica se debilitó sustancialmente debido a las presiones del mercado y por el acoplamiento de la educación superior a su lógica. (Borges, 2009).

\footnotetext{
${ }^{1}$ Este texto resulta de la investigación realizada en el ámbito del Doctorado en Educación realizado por la primera autora en la Universidad Estadual de Campinas, bajo orientación del segundo autor y coorientación del tercer autor.
} 


\section{LA REFORMA QUE LLEVÓ A LA PRIVATIZACIÓN DE LA EDUCACIÓN SUPERIOR EN BRASIL}

Como miembro de la OMC, Brasil, en el mandato del ex presidente FHC (1995 - 2002) emprendió una profunda reestructuración del Sistema Nacional de Educación Superior llevando a la privatización casi absoluta del sector.

El proyecto de reforma de este nivel de enseñanza, ejecutado en los ocho años de mandato de FHC, llevó al enjuagado de las universidades públicas con cortes radicales en las inversiones y, también, con la transferencia de la actividad de oferta de cursos y programas de educación superior al sector privado .

A lo largo de la década de 1990, se desencadenó una ampliación del espacio privado en las actividades directamente ligadas a la producción económica y también en el campo de los derechos sociales, conquistados por las luchas históricas de la clase obrera, lo que generó una profundización de la mercantilización de la educación, educación universitaria. Esta ampliación se realizó a través de dos movimientos: a) la expansión de las instituciones privadas, a través de la liberalización de los «servicios educativos»; b) la privatización interna de las universidades públicas, a través de las fundaciones de derecho privado, de los cobros de tasas y mensualidades por los cursos pagados y del establecimiento de alianzas entre las universidades públicas y las empresas, redireccionando las actividades de enseñanza, investigación y extensión. (Lima, 2011: 87).

Por el análisis, aunque preliminar, de los documentos de políticas públicas para la educación superior en Brasil se puede afirmar que a partir de 1990, el Gobierno siguió las directrices de la OMC y del BM para dirigir las políticas en los países periféricos a través de la publicación de informes y estudios. (Marinho, 2012, Simon, 2012) Entre ellos, destaco, a modo de ejemplo, «La enseñanza superior - las lecciones derivadas de la experiencia», publicado en 1994. En este son «sugeridas» las siguientes estrategias: a) diversificación de las instituciones de enseñanza superior y de los cursos; b) diversificación de las fuentes de financiación de las universidades públicas; y c) construcción de un nuevo marco político y jurídico que viabilizara la implantación de las directrices privatizantes de la educación. (Banco Mundial, 1994).

1994 - Educación superior: las lecciones de la experiencia - un texto que se afirma centrado en las políticas y prácticas para la reducción de la pobreza en el mundo en desarrollo. Promueve una asociación directa entre reducción de la pobreza y educación superior, al mismo tiempo que reduce esta última la formación, desde la foto de la portada, en la que está estampado un estudiante senegalés siguiendo atenta y solitario instrucciones escritas para montar un aparato. En el cuerpo del texto, hay indicaciones claras de la reforma pretendida: «la educación a distancia y los programas de aprendizaje abierto pueden aumentar, efectivamente ya bajo costo, el acceso de los grupos desfavorecidos» (página 33). Hay referencias a la eliminación de obstáculos como «movimientos estudiantiles fuertes y gobiernos débiles» (página 25), así como al «desarrollo de instituciones no universitarias y la financiación privada, con el fin de producir las cualificaciones exigidas por una economía de mercado» (p. 31), a ejemplo del Servicio Nacional de Aprendizaje Industrial (SENAI). Mientras que el título sugiere el pasado («experiencia») como la principal referencia, las «lecciones» se remiten al supuesto éxito prometido: cursos flexibles y de corta duración, bajo demanda. Lo que se pone en juego es el futuro próximo: «A menos que se implementen reformas para mejorar el desempeño de la educación superior, muchos países están destinados a entrar en el siglo XXI despreparados para competir en la economía global, en que el crecimiento estará cada vez más basado en el conocimiento técnico y científico «(página 25). (Barreto y Leher, 2008, página 425)

Y así se dio "el nacimiento» de la Reforma de la Educación Superior en Brasil, semejante al mito griego donde Atenea, la diosa de la sabiduría, habría nacido de la cabeza de Zeus, su padre, con la ayuda de Hefesto que partió la cabeza del dios del, Olympus.

A partir de 1997 una serie de instrumentos jurídicos se publicaron para reglamentar la Ley de Directrices y Bases de la Educación Nacional (LDB) n. 9.394 de 20 de diciembre de 1996, permitiendo así la reforma. La mayoría de estos fue en forma de Decreto, un instituto autoritario, ya que es sancionado por el Presidente de la República sin el análisis y votación en el Congreso Nacional. 
La LDB promovió una amplia diversificación del sistema de enseñanza superior, instituyó nuevas modalidades de cursos y programas, y estableció los fundamentos para la construcción de un sistema nacional de evaluación de la educación superior. Cuando a la naturaleza jurídica de las instituciones educativas, así esta ley dispone:

- Art. 19. Las instituciones de enseñanza de los diferentes niveles se clasificar en las siguientes categorías administrativas

- I públicas, así entendidas las creadas o incorporadas, mantenidas y administradas por el Poder Público;

- II privadas, así entendidas las mantenidas y administradas por personas físicas o jurídicas de derecho privado.

- Art. 20. Las instituciones privadas de enseñanza se encuadrar en las siguientes categorías. I particulares en sentido estricto, así entendidas las que son instituidas y mantenidas por una o más personas físicas o jurídicas de derecho privado que no presenten las características de los incisos abajo;

- II comunitarias, así entendidas las que son instituidas por grupos de personas físicas o por una o más personas jurídicas, incluso cooperativas educativas, sin fines de lucro, que incluyan en su entidad mantenedor a representantes de la comunidad,

- III confesionales, así entendidas las que son instituidas por grupos de personas físicas o por una o más personas jurídicas que atienden la orientación confesional e ideología específicas y lo dispuesto en el inciso anterior;

- IV filantrópicas, en la forma de la ley.

En cuanto a la educación superior en un capítulo dedicado exclusivamente a este nivel de enseñanza dispone la LDB:

- Art. 45 La educación superior será impartida en instituciones de enseñanza superior, públicas o privadas, con variados grados de alcance o especialización.

- Art. 52

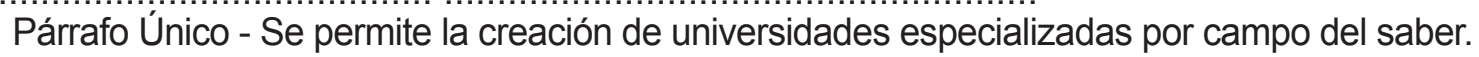

- Art. 54 - Las universidades mantenidas por el Poder Público gozarán, en la forma de la ley, de estatuto jurídico especial para atender a las peculiaridades de su estructura, organización y financiamiento por el Poder Público, así como de sus planes de carrera y del régimen jurídico de su personal.

Por lo que se refiere a la forma de organización de las entidades mantenedoras, de la organización académica de las instituciones universitarias privadas, se publicó el Decreto $n .^{\circ} 2.207$, de 5 de abril de 1997, regulando el Sistema Federal de Enseñanza.

- Artículo 1

Párrafo unico. Las entidades mantenedoras de las instituciones privadas de enseñanza superior podrán constituirse bajo cualquiera de las formas de persona jurídica, de derecho privado previstas en los incisos I y II del art. 16 del Código Civil Brasileño.

- Art. 4 - En cuanto a su organización académica, las instituciones de enseñanza superior del Sistema Federal de Enseñanza se clasificar en:

- I - universidades;

- II - centros universitarios;

- III - facultades integradas;

- IV - facultades;

- $\mathrm{V}$ - institutos superiores o escuelas superiores 
Sólo cuatro meses después de la publicación de este Decreto el mismo fue revocado por el Decreto n. ${ }^{\circ} 2.306$ de 19 de agosto de 1997, el foco aquí estaba en las atribuciones y la organización académica de las instituciones superiores privadas de enseñanza:

- Art. $1^{\circ}$ Las personas jurídicas de derecho privado, mantenedoras de instituciones de enseñanza superior, previstas en el inciso II del art. De conformidad con lo dispuesto en el artículo, de la Ley $N^{\circ}$ 9.394, de 20 de diciembre de 1996, podrán asumir cualquiera de las formas admitidas en derecho, de naturaleza civil o comercial y, cuando constituidas como fundaciones, se regirán por lo dispuesto en el art. 24 del Código Civil Brasileño.

- Art. $8^{\circ}$ En cuanto a su organización académica, las instituciones de enseñanza superior del Sistema Federal de Enseñanza se clasificar en:

- I Universidades;

- II Centros Universitarios;

- III Facultades Integradas;

- IV Facultades;

- V - Institutos Superiores o Escuelas superiores.

A partir de entonces el sector privado se expandió significativamente. Sin embargo, la consolidación de ese proceso se daría en 2001 con el Decreto n. ${ }^{\circ} 3.860$ de 09 de julio que revoca lo anterior. Una vez más las reglas de organización de la enseñanza superior han cambiado.

- Art. 7 - En cuanto a su organización académica, las instituciones de enseñanza superior del Sistema Federal de Enseñanza, se clasificar en:

- I Universidades;

- II Centros Universitarios;

- III Facultades Integradas;

- IV Facultades; Institutos Superiores y / o Escuelas superiores.

Se resalta que la naturaleza de las IES existentes no ha sido modificada, se han reagrupado a la luz del nuevo dispositivo legal.

Se puede afirmar que en la medida en que el sector privado se expandía el Gobierno alteraba los institutos jurídicos con el fin de facilitar este proceso. Pero hay quien califique esta asertiva como capciosa, especialmente los defensores del sector privado. Entonces se establece un debate que viene ocupando buena parte de las investigaciones y publicaciones acerca de las políticas públicas para la educación superior en Brasil a partir de la década de 1990.

Como ya dijimos, en el período 1995 a 2002 el número de instituciones el sector privado de educación superior creció mientras las públicas disminuyeron, conforme puede ser verificado en el cuadro abajo:

Evolución del Número de Instituciones por Dependencia Administrativa.

\begin{tabular}{|c|c|c|c|c|c|c|c|c|c|c|c|}
\hline Año & Total & \multicolumn{9}{|c|}{ Públicas } & \multicolumn{2}{c|}{ Privadas } \\
\hline & & Total & $\%$ & Federal & $\%$ & $\begin{array}{c}\text { Esta- } \\
\text { dual }\end{array}$ & $\%$ & Municipal & $\%$ & Privada & $\%$ \\
\hline 1995 & 894 & 210 & 23,4 & 57 & 27,1 & 76 & 36,1 & 77 & 36,6 & 684 & 76,5 \\
\hline 2002 & 1.637 & 195 & 11,9 & 73 & 37,4 & 65 & 33,3 & 57 & 29,2 & 1.442 & 88 \\
\hline$\%$ & 83,1 & $-7,1$ & - & $28 \%$ & - & $-14,4$ & - & $-25,9$ & - & 110,8 & - \\
\hline
\end{tabular}

Fuente: MEC/INEP/SEEC (1995) y MEC/INEP/DAES (2002). 
El crecimiento del sector fue del $83,1 \%$. Lo que demuestra la política privatista de este Gobierno fue que el número de instituciones privadas que creció el 110,8\% mientras que las públicas disminuyeron un $7,1 \%$. El descenso si yo entre las instituciones estatales y municipales. Estos datos nos permiten afirmar que la concepción de educación superior no fue considerada un bien público, en este gobierno. En el Gobierno Lula, a partir de 2003, la Reforma de la Educación Superior no fue interrumpida. El sistema pasó a la tercera fase de acuerdo con la clasificación de Trow (1973). En el Decreto Presidencial n. ${ }^{0} 5.225$ de 01 de octubre de 2004 los Centros Federales de Educación Tecnológica (CEFETs) se insertan en la estructura de la educación superior federal y se les otorga autonomía.

- Art. $11 \mathrm{~A}$-.....

- 10 Se extiende a los Centros Federales de Educación Tecnológica autonomía para crear, organizar y extinguir, en su sede, cursos y programas de educación superior dirigidos al área tecnológica, así como remanejar o ampliar plazas en los cursos existentes en esa área.

- $2^{\circ}$ Los Centros Federales de Educación Tecnológica podrán gozar de otras atribuciones de la autonomía universitaria, además de la que se refiere el §10, debidamente definidas en el acto de su acreditación, en los términos del $\S 2^{\circ}$ del art. 54 de la Ley no 9.394 de 1996.

Los CEFET ofrecerán Cursos Superiores de Tecnología (CST), estos son entendidos como graduación, enfocados en áreas específicas de conocimientos científicos y tecnológicos y se ofrecen en un tiempo menor que los cursos de bachillerato. A partir de la promulgación de este Decreto, los CST recibieron especial atención del sector privado. El Decreto Presidencial n. ${ }^{\circ} 5205$ de 20 de diciembre de 2004 regula las fundaciones de apoyo privadas dentro de las IFES. Este fue revocado por el Decreto n. ${ }^{\circ} 7.423$ del 30 de diciembre de 2010 que viene reglamentar de forma más detallada el objeto del decreto anterior. También en 2004, se creó el Programa Universidad para Todos (Prouni) que ofrece becas de estudio en instituciones privadas a estudiantes de bajos ingresos e instituye una política afirmativa en favor de negros e indígenas. La educación a distancia está regulada por el Decreto Presidencial n. ${ }^{\circ} 5.622$ de 19 de diciembre de 2005, una reivindicación antigua del sector privado. Este instrumento acabó por favorecer, también, la ampliación de las instituciones y cursos en esta modalidad de educación superior. En el último año de mandato del ex presidente Lula el siguiente escenario en el sector público y privado.

Evolución del Número de Instituiciones por Dependencia Administrativa.

\begin{tabular}{|c|c|c|c|c|c|c|c|c|c|c|c|}
\hline Año & Total & \multicolumn{7}{|c|}{ Públicas } & \multicolumn{2}{|c|}{ Privadas } \\
\hline & & Total & $\%$ & Federal & $\%$ & $\begin{array}{c}\text { Esta- } \\
\text { dual }\end{array}$ & $\%$ & Municipal & $\%$ & Privada & $\%$ \\
\hline 2002 & 1.637 & 195 & 11,9 & 73 & 37,4 & 65 & 33,3 & 57 & 29,2 & 1.442 & 88 \\
\hline & 2.378 & 278 & 11,6 & 99 & 35,6 & 108 & 38,8 & 71 & 25,5 & 2.100 & 88,3 \\
\hline & 45,2 & 45,5 & - & 35,6 & - & 66,1 & - & 24,5 & - & 45,6 & - \\
\hline
\end{tabular}

Fuente: MEC/INEP.

Como se puede ver en el cuadro anterior, las instituciones privadas corresponden al $88,3 \%$ del total en el último año del mandato del ex presidente Lula. El crecimiento en el período fue del $0,3 \%$. En cuanto a la constatación en el cuadro anterior, acerca del porcentaje de representatividad del sector privado, hay que atentar para el crecimiento de las instituciones públicas, del $45,5 \%$. 
Como vemos, el crecimiento de las instituciones públicas en el período fue expresivo; las federales crecieron $35,6 \%$, las estatales $66,1 \%$ y las municipales el $24,5 \%$.

Ante los datos arriba presentados se puede afirmar que el sector público tuvo atención especial del Gobierno Lula, mientras que en el Gobierno FHC fue secado.

\section{REFERENCIAS BIBLIOGRÁFICAS}

BANCO MUNDIAL. La enseñanza superior - Las lecciones derivadas de la experiencia. Washington, 1994. Avaiable from: http://www-wds.worldbank.org/external/default/WDSContentServer/WDSP/IB/2005/06/14/000090341_20050 614161209/Rendered/PDF/133500PAPER0Sp1rior0Box2150A1995001.pdf. Acessado em 9 de Jan. de 2016.

BARRETO, Raquel Goulart; LEHER, Roberto. Do discurso e das condicionalidades do Banco Mundial, a educação superior «emerge» terciária. Revista Brasileira de Educação, Rio de Janeiro, v. 13, n. 39, Dec. 2008. Available from <http:// www.scielo.br/scielo.php?script=sci_arttext\&pid=\$1413-24782008000300002\&lng=en\&nrm=iso >. Acessado em 12 Mar. 2015. http://dx.doi.org/10.1590/S1413-24782008000300002.

BORGES, Maria Creusa .de Araújo. A educação superior numa perspectiva comercial: a visão da Organização Mundial do Comércio. RBPAE. v.25, n.1, p. 83-91, jan./abr. 2009.

BRASIL. Lei n. ${ }^{\circ}$ 9.394, de 20 de dezembro de 1996. Estabelece as Diretrizes e Bases da Educação Nacional.

Decreto 2.207, de 15 de abril de 1997. Regulamenta, para o Sistema Federal de Ensino, as disposições contidas nos arts. $19,20,45,46$ e $\S 1^{\circ}, 52$, parágrafo único, 54 e 88 da Lei n. ${ }^{\circ} 9.394$, de 20 de dezembro de 1996, e dá outras providências.

Decreto 2.306 de 19 de agosto de 1997. Regulamenta, para o Sistema Federal de Ensino, as disposições contidas no art. 10 da Medida Provisória n. ${ }^{\circ} 1.477-39$, de 8 de agosto de 1997, e nos arts. 16, 19, 20, 45, 46 e $\S 1^{\circ}$, 52, parágrafo único, 54 e 88 da Lei n. ${ }^{\circ} 9.394$, de 20 de dezembro de 1996, e dá outras providências.

LAMPREIA, Luiz Felipe Palmeira. Resultados da Rodada Uruguai: uma tentativa de síntese.

Estud. av., São Paulo, v. 9, n. 23, p. 247-260,Apr. 1995. Available from <http://www.scielo.br/scielo.php?script=sci_arttext\&pid=S0103- 40141995000100016\&lng=en\&nrm=iso>. access on 13 Feb. 2016.http://dx.doi.org/10.1590/S010340141995000100016.

LIMA, Licínio; AFONSO, Almerindo Janela. (2002). Reformas da educação pública: Democratização, modernização, neoliberalismo. Porto: Afrontamentos.

MARINHO, Claisy; POLIDORI. Marlis; ONO, Nathan. Democratização e expansão da educação superior no Brasil. In: LEITE, Denise; FERNANDES, Cleoni Barboza (Orgs.); BROILO, Cecília Luiza (Colab.). (2012). Qualidade da educação superior: Avaliação e implicações para o futuro da universidade. Porto Alegre: EDIPUCRS, p. 278 - 284. 
SIMON, Marinice Souza; MOROSINI, Marília Costa. Olhar crítico sobre a avaliação do ensino superior no Brasil. In: LEITE, Denise; FERNANDES, Cleoni Barboza (Orgs.); BROILO, Cecília Luiza (Colab.) (2012). Qualidade da educação superior: Avaliação e implicações para o futuro da universidade. Porto Alegre: EDIPUCRS, p. 109 - 115.

TROW, Martin. (2005) Reflections on the Transition from Elite to Mass to Universal Access: Forms and Phases of Higher Education in Modern Societies since WWII. Forthcoming in Philip Altbach, ed., International Handbook of Higher Education, Kluwer. 